\section{Cahiers de Narratologie}

Analyse et théorie narratives

34 | 2018

Les avatars du chapitre en bande dessinée

\title{
Jacques Tardi ou le parti pris de la continuité
}

\section{Thierry Groensteen}

\section{(2) OpenEdition}

\section{Journals}

Electronic version

URL: http://journals.openedition.org/narratologie/8669

DOI: 10.4000/narratologie.8669

ISSN: 1765-307X

\section{Publisher}

LIRCES

\section{Electronic reference}

Thierry Groensteen, « Jacques Tardi ou le parti pris de la continuité », Cahiers de Narratologie [Online], 34 | 2018, Online since 21 December 2018, connection on 15 November 2019. URL : http:// journals.openedition.org/narratologie/8669; DOI : 10.4000/narratologie.8669

This text was automatically generated on 15 November 2019.

Article L.111-1 du Code de la propriété intellectuelle. 


\title{
Jacques Tardi ou le parti pris de la continuité
}

\author{
Thierry Groensteen
}

1 Ici Même est le premier roman graphique dessiné par Jacques Tardi, si l'on entend par roman graphique une bande dessinée dont l'ampleur dépasse celle des albums de la tradition franco-belge, les fameux « $48 \mathrm{CC}$ ». Or, Ici Même, comme l'on sait, n'est pas un scénario de Tardi. Pour la quatrième fois dans son encore jeune carrière (après Rumeurs sur le Rouergue, Griffu et Polonius), le créateur d'Adèle Blanc-Sec s'était associé à un scénariste, en l'espèce Jean-Claude Forest.

2 Ici Même est une bande phare du nouveau mensuel (A Suivre), puisqu'elle est choisie pour faire la couverture du premier numéro, en février 1978. Elle en réalise exemplairement le programme, à savoir l'introduction d'une "densité romanesque " dans la bande dessinée - pour reprendre les mots employés par Jean-Paul Mougin, le rédacteur en chef, dans son premier éditorial. Pourtant, selon le témoignage de Tardi, Forest et lui avaient commencé à travailler sur cette histoire avant d'être contactés par (A Suivre), sans trop savoir à qui ils iraient la proposer (Finet $2004: 28$ ). Ils se sont donc, par chance, trouvés en phase avec le concept du nouveau magazine lancé par Casterman; ils avaient un projet tout prêt répondant parfaitement au cahier des charges.

3 L'album paraît à l'automne 1979 et inaugure la collection des « Romans (A Suivre)»; celle-ci, comme l'on sait, avait été conçue sur le modèle de La Ballade de la mer salée, d'Hugo Pratt, qui, selon l'éditeur Didier Platteau « incarnait un peu le prototype de ce que nous voulions faire» (Finet $2004:$ 15). Par une troublante coïncidence, Ici Même totalise 163 pages, soit très exactement le même nombre que La Ballade. Avec cette différence que, si l'album de La Ballade n'est pas chapitré, Ici Même, en revanche, l'est.

4 La prépublication par fragments de l'œuvre de Tardi et Forest dans (A Suivre) n'explique pas tout. En effet $L a$ Ballade avait elle aussi connu une prépublication initiale, en Italie, dans le mensuel Sgt Kirk, de juillet 1967 à février 1969, du n 1 au n 20 (avec des interruptions aux $n^{\circ} 2,3$ et 16) - en dix-sept épisodes donc. Il faut imputer la division en chapitres à un choix concerté des auteurs. Des auteurs ou, plus vraisemblablement, 
du scénariste. En effet, Forest a expliqué : «Je crois que sans ces articulations, le lecteur se fatiguerait plus vite et se perdrait dans l'histoire. Il doit être content de tomber de temps en temps sur une page blanche" (Groensteen 1980: 110). Dans le même entretien, qu'il m'avait accordé en 1980, il révélait que Tardi, surchargé de travail à un moment, n'avait pas pu tenir le rythme prévu, et que, des deux derniers chapitres, ils avaient dû en faire trois.

D'autres œuvres de Forest confirment que le scénariste d'Ici Même était un adepte de la division du récit en chapitres. La Jonque fantôme vue de l'orchestre (1981), bien sûr, qui en compte huit, mais déjà des ouvrages antérieurs à l'ère (A Suivre) : Mystérieuse matin midi et soir avait été publié dix ans avant la Jonque, et dans Pif Gadget. Deux épisodes avaient paru dans l'hebdomadaire, en avril et mai 1971. La rédaction n'avait pas laissé paraître le troisième, mais l'album, lui ${ }^{1}$, est bien divisé en trois parties numérotées, pourvues, chacune, d'un titre. Dans le premier Barbarella, prépublié dans V-Magazine, les " chapitres » sont plus courts et ne sont pas aussi marqués, ni revendiqués comme tels. Mais le livre reprend la bichromie en vigueur dans le magazine, avec la deuxième couleur qui change d'une séquence à l'autre. Le lecteur parcourt cinq ambiances colorées distinctes, qui scandent et périodisent les 65 planches du récit.

On pourrait penser que le chapitrage est l'indice d'une ambition littéraire. Cependant d'autres éléments peuvent charger une bande dessinée en littérarité, et ils ne sont pas nécessairement corrélés avec celui-là. J'ai dit que $L a$ Ballade de la mer salée n'est pas chapitrée. Pourtant Pratt revendique l'influence des grands écrivains de la mer et du roman d'aventures en général, et son récit est truffé de références littéraires,

tantôt discrètes, tantôt plus appuyées, au Voyage autour du monde de Bougainville (p.

$8^{2}$ ), à Robinson Crusoé (p. 34), à Moby Dick (p. 54 ; le visage de Tarao rappelle, à

l'évidence, celui du harponneur Quiequeg), à Coleridge (p. 60), aux poètes Rilke et

Shelley (p. 161), à Euripide enfin (p. 166). (Groensteen $2015: 32$ )

7 Un autre indice de littérarité est le recours à un narrateur (ou " récitant ») dont la voix porte le récit, assurant notamment l'ancrage des séquences dans le temps et dans l'espace. Or, l'un des éléments frappants de la poétique mise en œuvre dans Ici Même est le fait que le récit ne compte aucun récitatif, aucune indication temporelle, aucune mention verbale qui articulerait les scènes entre elles : la narration est tout entière au présent, en direct, sans intercesseur ni énonciateur explicite.

8 Ce qui, en revanche, peut être regardé comme un fait littéraire dans l'œuvre conjointe de Tardi et Forest, c'est la langue. Une langue très écrite, des phrases comprenant souvent des parties entre tirets ou entre parenthèses. Dans cette ambiguïté constitutive du dialogue de bande dessinée, qui participe toujours à la fois de l'oralité et de l'écriture, il est clair que Forest a choisi de pencher du côté de l'écrit. La chose n'est pas surprenante quand on connaît la méthode de travail qui était la sienne. En réponse à la question "de quelle manière travaillez-vous quand vous écrivez un scénario ", il répondait :

Au départ, j'écris toujours un synopsis copieux, presque un petit roman, dans lequel je fourre tout ce qui me vient à l'esprit: bribes de dialogues, indications de décor, etc. Ensuite je fais le découpage chapitre par chapitre, au fur et à mesure que la réalisation graphique progresse. (Groensteen \& Peeters $1986: 108$ )

Chapitre ne doit pas être entendu ici au pied de la lettre, Forest aurait sans doute pu dire séquence. Ce qui importe, ici, c'est le fait que ses ouvrages connaissent d'abord une 
forme écrite, littéraire, et que l'adaptation qui en est faite ensuite pour le médium bande dessinée conserve la trace de cette origine.

Ici Même représentait, pour Tardi, le premier travail d'aussi longue haleine, le premier album de plus de 100 pages. Dans la suite de sa carrière, il va publier de nombreux autres romans graphiques, dont certains seront plus épais encore que celui-là : 184 pages pour 120 rue de la gare, 188 et 238 pages pour les tomes 1 et 2 de Moi, René Tardi, prisonnier de guerre au Stalag IIB. Or, sauf exception - nous verrons le détail dans un instant -, Tardi n'a pas conservé le modèle forestien, il n'est pas resté fidèle au chapitrage. Il n'y a pas de chapitres à l'intérieur des deux volumes de Moi, René Tardi, il n'y en a pas non plus dans Brouillard au pont de Tolbiac, et les quatre volumes du Cri du peuple, qui comptent environ 80 pages chacun, sont, eux aussi, d'un seul tenant.

11 Pourtant, le chapitrage va s'imposer, sinon comme la règle, en tout cas comme l'usage dominant chez les auteurs alimentant la collection des « romans (A Suivre) »: Forest, bien sûr, avec La Jonque fantôme (divisé, je l'ai dit, en huit chapitres), mais également Comès avec Silence (huit chapitres également), F’Murr avec Jehanne au pied du mur (un prologue et quatre chapitres), Schuiten et Peeters avec les différents volumes des Cités obscures, ou encore Muñoz et Sampayo dans les différents volumes de la série Alack Sinner, pour ne citer que ceux-là...

En règle générale, Tardi semble, quant à lui, vouloir privilégier l'immersion du lecteur dans un flux narratif que rien ne vient interrompre. Cela peut, du reste, être une stratégie narrative dictée par le sujet, en adéquation avec la nature même du récit. Ainsi du long périple du père de Tardi et de ses camarades prisonniers à travers l'Europe en guerre, tel que le reconstitue le deuxième tome de Moi, René Tardi... : on peut penser que le fait de laisser le récit couler sans interruption souligne le caractère interminable et harassant de cette marche dont l'intéressé ne voyait pas la fin.

13 Cependant, il semble bien que l'on puisse parler d'une réticence, voire d'une hostilité de principe de Tardi à l'endroit de la segmentation. En effet, lorsqu'il adapte un roman, il supprime les chapitres s'il le peut. Prenons 120, rue de la Gare. Le roman de Léo Malet, paru en 1943, était chapitré. L'album qu'en a tiré Tardi (1988) ne l'est pas. Dans son « autopsie » de cette adaptation, Jean-François Douvry observe :

Le roman de Malet comporte un prologue (Allemagne) et deux parties (Lyon, puis Paris), subdivisées en chapitres. Tardi conserve la structure générale, y compris dans la place attribuée à chacun des trois lieux principaux, mais il réduit considérablement le nombre de chapitres, dont il change les titres. (Douvry 1988 :

23)

Douvry fait référence à la prépublication, en quinze livraisons, dans (A Suivre) - avec une interruption de six mois entre la première et la deuxième moitié du récit ${ }^{3}$; les " chapitres " y portaient des titres tels que "Cinq détectives dans la ville », « Le Père Noël est en avance ", "L'ombre de Jo Tour Eiffel » ou encore "Un réveillon plein d'imprévu », et ils étaient introduits par une page de titre, conformément à l'usage du périodique. Mais aucun de ces titres ne se retrouve dans l'album, où les différentes séquences s'enchaînent sans rupture, sans aucune scansion marquée.

Pour les autres romans de Malet qu'il adaptera (Brouillard au pont de Tolbiac en 1982, six ans avant 120, rue de la Gare; ou Casse-Pipe à la Nation, pour ne citer que les principaux), Tardi effacera de même toute trace du découpage de l'intrigue en chapitres conçu par l'écrivain. En revanche, dans Jeux pour mourir, adapté d'un autre roman policier, de GéoCharles Véran, la division en quatre chapitres est conservée. Si l'album est très épais 
(238 pages), cela tient en partie au fait que les planches ne comptent pas plus de trois ou quatre images. Ici, la structure quaternaire épouse l'action, divisée en quatre journées consécutives, les $26,27,28$ et 29 août 1950 - sans compter que les assassins, représentés en couverture, sont eux aussi au nombre de quatre. Tardi introduit chaque chapitre par une mention de la date, à la façon d'un calendrier, sur fond blanc et en "belle page ». Curieusement, il reprend systématiquement la date dans un cartouche intégré à la première case du chapitre même. Cette mention fait double emploi et peut laisser penser que, dans un premier temps, Tardi avait choisi de se contenter d'elle mais que, par la suite, il s'est rendu à l'idée que les quatre parties méritaient une ouverture plus nette - sans modifier ses planches pour autant.

Il est certain que, pour le coup, le non-respect de la périodisation du récit aurait nui à la compréhension de l'intrigue. $\mathrm{Au}$ reste, le sentiment du temps qui s'écoule est généralement essentiel à la mécanique d'un récit policier ; écoulement fatal quand il précède un meurtre, empreint d'un sentiment d'urgence quand c'est le temps de l'enquête.

17 Il est intéressant d'observer que, dans Brouillard au pont de Tolbiac, la perception du temps est rendue beaucoup plus incertaine. Le récit s'ouvre sur la date du 10 novembre 1956 (première planche, p. 9), puis un flash-back de trois pages nous déporte vers le 15 décembre 1927 (page 19) et, après le retour à l'action principale, il faut attendre la page 48 pour trouver une nouvelle indication temporelle («Le lendemain... »), dont le lecteur ne saurait dire avec certitude si elle correspond à la date du 11 novembre. À la page 67, un nouvel énoncé donne à comprendre qu'une nouvelle nuit est passée : «Il était $14 \mathrm{~h}$. Depuis $2 \mathrm{~h}$ du matin, heure à laquelle j'étais rentré chez moi... » Enfin, page 74, à une page de la fin de l'histoire, on lit «Vingt-quatre heures plus tard... » mais, à ce moment-là, il y a beau temps que les coordonnées temporelles précises de l'intrigue se sont effacées de la mémoire du lecteur.

18 Ce qui contribue à rendre la perception du temps confuse, c'est que, si le récit que Burma nous livre de son enquête est entièrement au passé, l'album comporte quelques récitatifs au présent, imputables à ce que j'appellerai le narrateur principal (ou le récitant extradiégétique), toujours corrélés à la même vue récurrente. Ainsi de la case inaugurale : "PARIS. La nuit, sur le pont de Tolbiac, un homme rôde. Dans son regard, la folie ", case dont le statut est incertain puisque Burma n'assiste pas à cette scène et n'en a pas connaissance. Une reprise de cette image, toujours aussi étrangère au récit principal, et dans un cadrage toutefois différent, survient page 35, accompagnée d'un texte presque identique : «Au même instant, sur le pont de Tolbiac, un homme rôde. Dans son regard, la folie. " Mais cette fois le au même instant établit la concomitance entre l'errance de cet homme mystérieux et la scène impliquant Burma présentée sur la même page. De fait, les deux récits se rejoignent à la page 44 , quand Burma découvre le cadavre de l'homme en question, qui a été poignardé (une coupure de presse reproduite quatre pages plus loin établira, enfin, son identité). Ainsi le présent et le passé, le récit impersonnel du narrateur et celui délivré par le protagoniste ont convergé, mais non sans avoir provisoirement quelque peu égaré le lecteur.

En réalité, Tardi est beaucoup plus préoccupé des déplacements accomplis par son héros dans le tissu urbain du 13e arrondissement. Ses trajets sont dûment répertoriés sur une carte donnée en annexe, sur une double page. De même, le diptyque Moi, René Tardi est borné par deux cartes: au début du tome 1, celle de tous les stalags et oflags créés par les Allemands durant la Seconde Guerre mondiale, d'où se détachent, en 
rouge, ceux qui serviront de décor à l'action; à la fin du tome 2, le tracé de la longue marche du père de l'auteur lors de son retour en France.

On sait que Tardi a toujours accordé une très grande importance aux repérages, à la vérification scrupuleuse par lui-même des lieux qu'il serait appelé à représenter. La vérité du lieu est pour lui, paradoxalement, la condition d'une certaine liberté dans la conduite du récit et les choix de mise en scène. Mon hypothèse est que la relative indifférence de Tardi vis-à-vis du découpage temporel de ses récits est l'envers de son attachement à leur ancrage spatial.

Penchons-nous de plus près sur la prépublication de C'était la guerre des tranchées. La première livraison paraît en mars 1982 dans (A Suivre) n ${ }^{\circ} 50$ et compte 20 planches. Trois autres livraisons suivent dans les numéros 53 (juin 1982), 54 (juillet) et 58 (novembre), qui comptent respectivement 7, 10 et 14 planches. Puis, pendant dix ans, rien. Aucune explication donnée au lecteur. Tardi semble avoir définitivement délaissé une œuvre qui s'annonçait pourtant d'une force exceptionnelle. Les 51 planches existantes ne sont pas rassemblées. Au cours de cette période, Tardi dessine notamment un Adèle Blanc-Sec, trois Nestor Burma, Tueur de cafards et Jeux pour mourir. Puis, de façon inattendue, la Guerre des tranchées revient dans (A Suivre) en février 1993, au $n^{\circ} 181$, avec 14 nouvelles planches. Les deux derniers épisodes paraîtront dans les numéros 185 (juin) et 189 (octobre) et compteront respectivement 12 et 15 planches. L'album, lui, est introduit en librairie en octobre 1993, en même temps que les dernières planches paraissent dans le mensuel.

Les raisons exactes de cette très longue interruption ne me sont pas connues. Dans ( $A$ Suivre), les pages de titre des sept livraisons que l'on retrouvera dans l'album étaient toutes identiques : une décoration militaire ensanglantée accompagnée d'une citation patriotique ayant, dans le contexte de cette bande dessinée, valeur d'antiphrase ironique (fig. 1). Seule la mise en page de ces pages de titre a légèrement évolué entre 1982 et 1993, avec l'introduction de la couleur rouge sang. Dans l'album, ces têtes de chapitre ont évidemment sauté (et avec elles les citations, ce que l'on peut considérer comme une perte); mais le motif de la médaille ensanglantée revient, en noir et blanc, sur la page de titre, et en couleur sur la quatrième de couverture. 


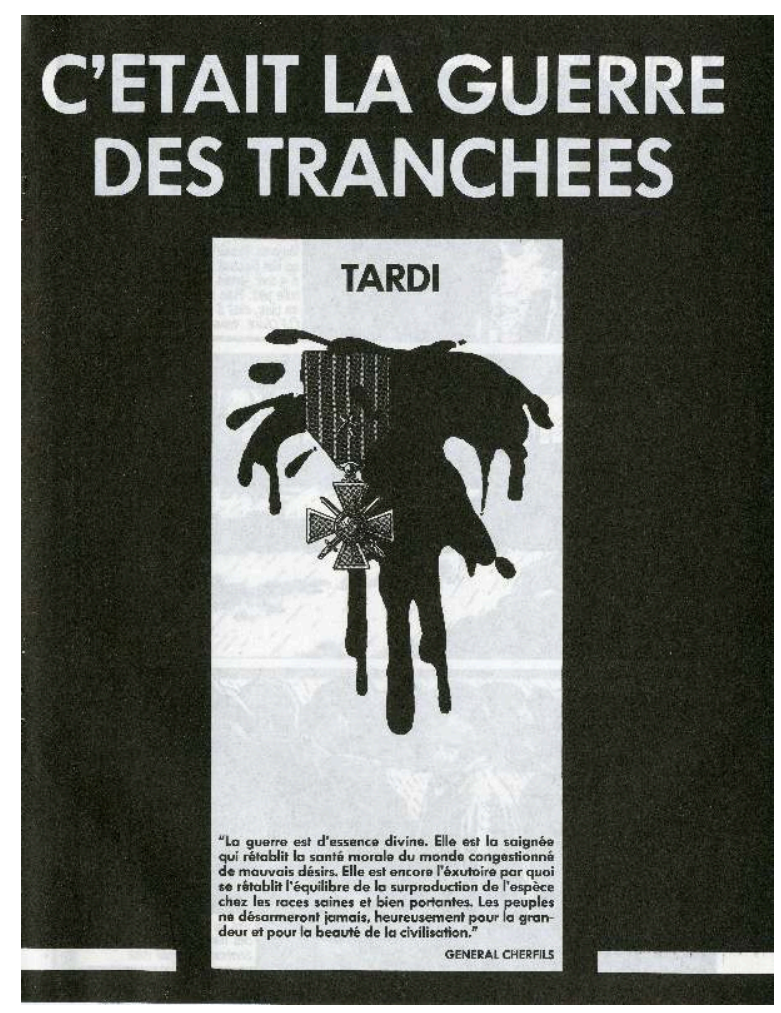

Figure 1. Revue (A Suivre) n53, page de titre du chapitre de Tardi, @ Casterman.

Avec l'aimable autorisation des auteurs et des Éditions Casterman.

L'album s'émancipe des contraintes et des aléas de la prépublication et trouve sa propre forme. Il obéit à une logique différente et lui seul peut se réclamer des conceptions de l'auteur. S'agissant de C'était la guerre des tranchées, Tardi a recyclé, au début de l'ouvrage, les 20 planches du Trou d'obus, un album paru en 1984 sous la marque de l'Imagerie Pellerin. Sur le plan de la mise en page, les planches du Trou d'obus ne respectent pourtant pas l'immuable structure ternaire, la division en trois bandes panoramiques d'égale dimension, caractéristiques de toutes les planches parues dans (A Suivre). Seule la communauté de thème justifie cette reprise, et la volonté de donner une carrière plus large à un récit demeuré jusque-là relativement confidentiel. Le Trou d'obus sert donc de prologue à C'était la guerre des tranchées, séparé du reste de l'ouvrage par deux pages de texte.

Bien que les sept « chapitres » ne soient plus indiqués comme tels ni séparés d'aucune manière, est-ce que lecteur continue de percevoir plus ou moins consciemment cette division originelle? Il faudrait, pour cela, que les chapitres constituent des épisodes plus ou moins clos sur eux-mêmes, avec une unité de sujet ou un effet de clôture. L'examen des planches le confirme en partie. On peut, en particulier, considérer que les planches correspondantes, lors de la prépublication, aux quatrième, cinquième et sixième livraisons, présentent bien les caractéristiques d'un épisode cohérent, identifiable comme tel, en raison des indications temporelles qui les introduisent : « 29 août 1914 », «Janvier 1916 » puis « Janvier 1918 ». Ces trois épisodes sont ancrés dans une chronologie précise des faits et sont séparés par de longs intervalles, par des ellipses qui assurent leur disjonction. L'entrée dans le septième et dernier « chapitre » virtuel est moins marquée. Cette fois, la datation des faits rapportés intervient, non au début 
de l'épisode, mais à la fin, dans la dernière page du récit, dans la dernière case même, qui porte la mention de la date de l'armistice, le 11 novembre 1918.

En dehors de ces marqueurs explicites que sont les inscriptions temporelles, d'autres éléments peuvent assurer la clôture d'un épisode. S'agissant du sixième pseudochapitre, celui daté « janvier 1918 », on peut observer que la case d'ouverture (page 100 de l'édition originale) se trouve reprise dans la douzième et dernière planche de cette même séquence, non pas tout à fait en position terminale mais avant-dernière. Il y a là un effet de boucle, qui ressortit à ce que j'appelle le tressage, et qui constitue lui aussi un indice de l'homogénéité de l'épisode, nous invitant à le lire comme tel.

Il faut toutefois prendre garde que de semblables « rimes » peuvent s'observer ailleurs qu'en situation de borner un épisode. Ainsi, la quatrième livraison s'achevait par une case montrant le profil du soldat Mazure. Or, cette case renvoie bien à une autre, vue antérieurement, mais cette image correspondante se situe quatre pages en amont et ne constitue pas l'autre borne de l'épisode, commencé encore neuf pages plus haut. Ici, la rime (pages 81 et 85 ) ne nous signale pas l'entrée et la sortie d'un chapitre implicite (fig. 2 et 3). En revanche, les 14 planches de cette livraison possèdent une unité forte sur le plan du contenu narratif, puisqu'elles sont entièrement centrées sur la personne du soldat Mazure, successivement planqué, fait prisonnier, libéré et finalement condamné pour abandon de poste devant l'ennemi. La Guerre des tranchées n'est pas un récit linéaire mais une collection de scènes plus ou moins indépendantes les unes des autres; plus qu'un album déroulant une intrigue classique, cet ouvrage est donc, dans sa conception même, structuré par un chapitrage implicite.
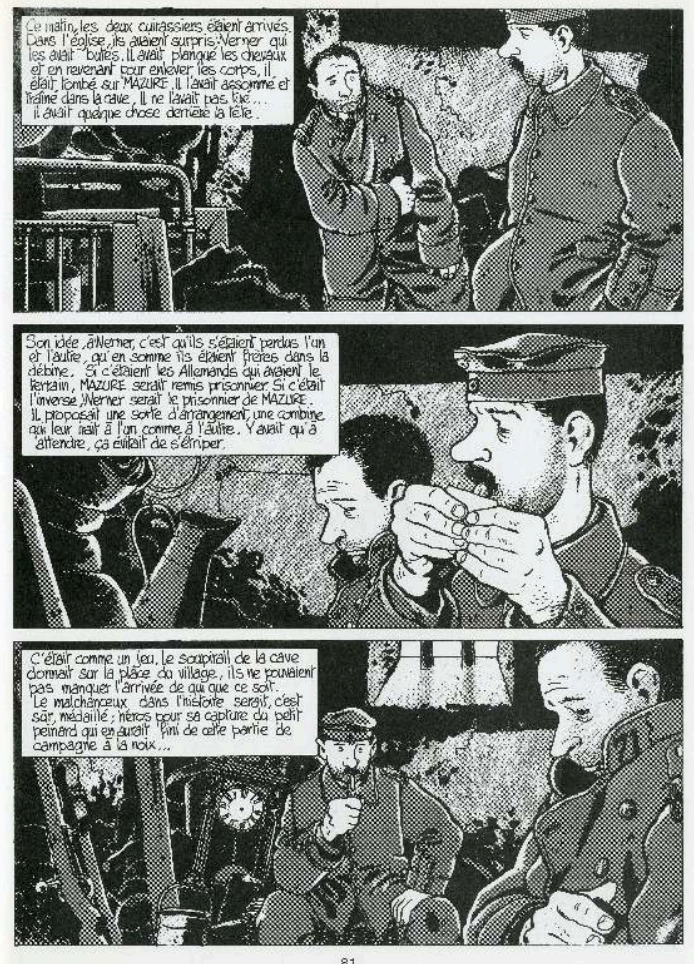

Figure 2. Pages 81 C'était la guerre des tranchées, de Tardi (1993) @ Casterman. Avec l'aimable autorisation des auteurs et des Éditions Casterman. 


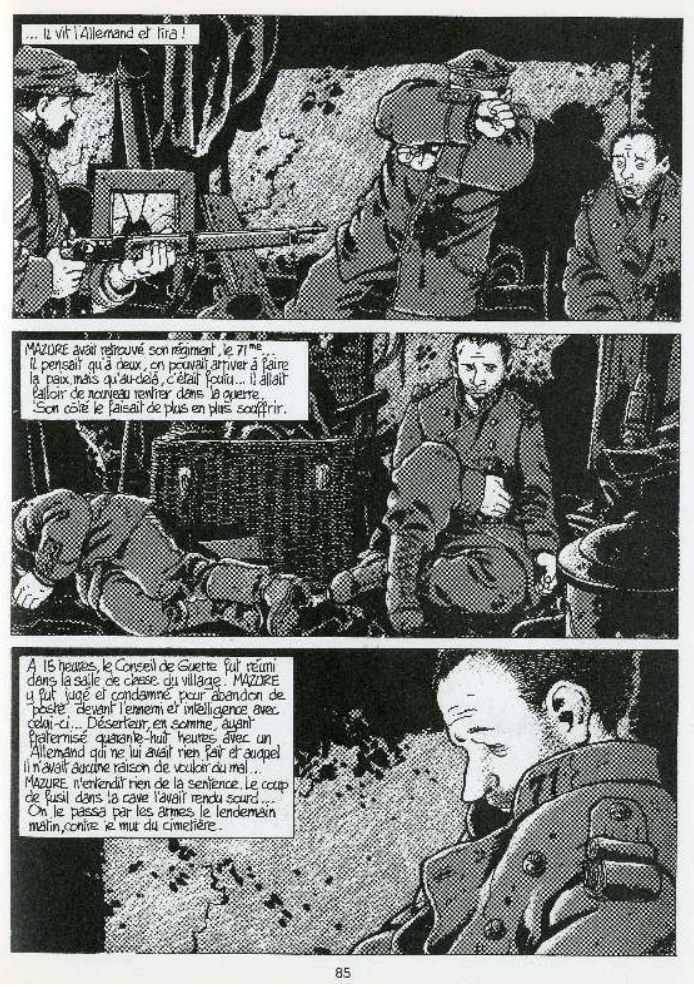

Figure 3. Page 85, C'était la guerre des tranchées, de Tardi (1993) @ Casterman. Avec l'aimable autorisation des auteurs et des Éditions Casterman.

Lors de la prépublication dans un mensuel, en revanche, il n'est pas toujours possible de savoir si l'interruption de l'épisode à tel endroit correspond à une scansion volontaire dans la narration, ou à un impératif rédactionnel (il n'y avait pas plus de pages disponibles dans ce numéro) ou encore au fait que le dessinateur n'a pas eu le temps de livrer davantage de planches. Au début de sa carrière, Tardi déclarait :

J'aimais bien la formule de l'hebdomadaire avec l'histoire à suivre qui durait six mois. Je retombe peut-être dans une nostalgie de gosse qui était abonné à Spirou ou Tintin, mais je trouvais ça passionnant de suivre chaque semaine mon héros favori. Maintenant, on fait des mensuels ou pis, des trimestriels qui cassent complètement la formule de l'histoire à suivre. Pour pallier cet inconvénient, on passe carrément dix à quinze pages à la fois, et tout l'intérêt de l'attente propre au roman feuilleton est complètement saccagé. Je crois que la $\mathrm{BD}$ accédera vraiment à l'âge adulte quand on pourra, comme en littérature, sortir une histoire directement en album. (Groensteen 1980 : 115)

Un album qui ne ressemblerait pas à un recueil mais bien à un roman. On peut dire que l'essor du « roman graphique » est venu exaucer les vœux de Tardi et lui a permis de concrétiser ses ambitions.

Si l'album consiste en un regroupement de pseudo-chapitres dispersés dans les livraisons successives d'un même magazine, et s'il efface les traces de ce morcellement initial, le même phénomène peut se reproduire identiquement lorsqu'une série d'albums se voient regroupés au sein d'une intégrale en un seul épais volume. C'est ce qui s'est passé pour Le Cri du peuple. Les quatre tomes, d'environ 80 pages chacun, dont la publication s'était échelonnée de 2001 à 2004, ont fusionné dans un seul gros volume en 2005. On aurait pu penser que les quatre volumes fourniraient un découpage naturel en quatre parties - d'autant qu'ils étaient dotés de titres qui les autonomisaient, 
respectivement "Les canons du 18 mars", "L'espoir assassiné », "Les heures sanglantes » et "Le testament des ruines». Mais ce n'est pas le cas: au contraire, l'intégrale ne comporte aucune forme de périodisation du récit et propose plus de 300 planches d'une seule traite. Seules ont été supprimées les planches qui interrompaient arbitrairement chacun des tomes initiaux par une série de questions relevant de la rhétorique feuilletonesque chère à l'auteur des Aventures extraordinaires d'Adèle Blanc-Sec ; les autres pages s'enchaînent sans heurt et sans qu'il ait été besoin de les retoucher. Le destin éditorial d'une œuvre, le passage de la prépublication à l'édition sous la forme d'un livre, ou de la publication en plusieurs volumes à celle d'une intégrale, oblige à considérer la question du chapitrage dans une perspective diachronique et évolutive. Chez Tardi, il est clair que, sauf quand d'impérieuses raisons narratives obligent à maintenir un découpage en parties identifiées comme telles, la pente naturelle de l'auteur est de supprimer toute forme de séquençage au profit d'un récit complètement intégré, homogène, au déroulement continu, et de laisser au lecteur le soin d'interrompre sa lecture où et quand il le souhaite, de lui confier la maîtrise du rythme, le choix des étapes.

\section{BIBLIOGRAPHY}

Douvry, Jean-François (1988) Rendez-vous... 120, rue de la gare : autopsie d'une adaptation, Paris, Casterman.

Finet, Nicolas (2004), (A Suivre) 1978-1997 : une aventure en bandes dessinées, Paris, Casterman.

Groensteen, Thierry (1980), Tardi, monographie, Bruxelles, Magic Strip.

Groensteen, Thierry (2015), Un art en expansion, Bruxelles, Les Impressions Nouvelles.

Groensteen, Thierry \& Benoît Peeters (1986), « Jacques Lob - Jean-Claude Forest, entretien », Revue de l'Université de Bruxelles, $\mathrm{n}^{\circ}$ 1-2, p. 103-109.

\section{NOTES}

1. Mystérieuse matin midi et soir (SERG, 1973 ; réédité par l'Association en 2004).

2. La pagination se réfère à l'édition originale, parue chez Casterman en 1975.

3. Numéros 97 à 105 (février-octobre 1986) et numéros 112 à 117 (mai-octobre 1987), avec un résumé des épisodes précédents dans le $\mathrm{n}^{\circ} 112$. 


\section{ABSTRACTS}

Ici Même, the first graphic novel drawn by Jacques Tardi (on a script by Jean-Claude Forest), was divided in chapters, indicating the serialization in the monthly magazine (A Suivre). In his later works, however, including Moi, René Tardi, prisonnier de guerre au Stalag IIB, Tardi, against the practice that becomes common for the comic narratives of a certain length, will refuse as much as possible the principle of chapters, prefering to submerging his readers in a continuity, an uninterrupted flow. Nevertheless, certain evidences of distinct sections can be observed in C'était la guerre des tranchées and, more obviously, in Jeux pour mourir.

Premier roman graphique dessiné par Jacques Tardi, Ici Même (scénario : Jean-Claude Forest) était divisé en chapitres, témoignant de la sérialisation dans le mensuel (A Suivre). Par la suite, en revanche, et jusqu'à Moi, René Tardi, prisonnier de guerre au Stalag IIB, Tardi, à rebours de l'usage qui tend à se répandre pour les œuvres de bande dessinée d'une certaine ampleur, se dérobera autant que possible au principe du chapitrage, préférant immerger son lecteur dans une continuité narrative, un flux ininterrompu. Des traces de périodisation du récit peuvent néanmoins être repérées dans C'était la guerre des tranchées et, plus explicitement, dans Jeux pour mourir.

\section{INDEX}

Keywords: «(A Suivre) », chapters, Jacques Tardi, serialization, graphic novel, subdivision, breading

Mots-clés: « (A Suivre) », chapitres, Jacques Tardi, prépublication, roman graphique, séquençage, tressage

\section{AUTHOR}

\section{THIERRY GROENSTEEN}

Thierry Groensteen est chargé de mission à la Cité internationale de la bande dessinée et de l'image, directeur de collection chez Actes Sud et rédacteur en chef de la revue en ligne NeuvièmeArt2.0. Il intervient régulièrement à l'École européenne supérieure de l'image. Il est l'auteur d'une vingtaine d'ouvrages, parmi lesquels Système de la bande dessinée (PUF, 1999), Un objet culturel non identifié (L'An 2, 2006) et Un art en expansion (Les Impressions nouvelles, 2015). Site : www.editionsdelan2.com/groensteen; courriel : thierry.groensteen@wanadoo.fr 